Military Technical College Kobry El-Kobbah, Cairo, Egypt

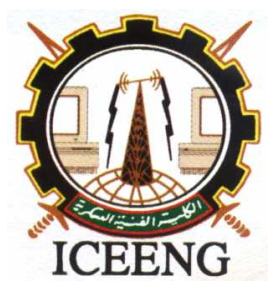

\author{
$6^{\text {th }}$ International Conference \\ on Electrical Engineering \\ ICEENG 2008
}

\title{
Application of modified covariance algorithm in state estimation
}

By

Nursyarizal Mohd Nor*
Prof. Dr Ramiah Jegatheesan**

\section{$\underline{\text { Abstract: }}$}

This paper describes an approach to identify and change the measurement weights used in Weight Least Square (WLS) estimation method employed in State Estimation (SE). In practice, the individual measurement is assigned with their own weight factor based on technical experience by the engineers. However, uncertainty in analog measurements could occur in a real time system. Those measurements that are assigned with high weighting factor need not be a good data. The method of Modified covariance (MC) proposed in this paper can identify and change the measurement weights of the bad measurements if the related measurement is assigned with high weight factors. Simulated measurement data and state estimation results are used as historical data. The proposed method is tested on IEEE 24-bus network and SE provides reliable estimation.

\section{Keywords:}

State Estimation (SE), Weight Least Square (WLS), Autoregressive (AR), Modified Covariance (MC) algorithm.

* Electrical and Electronics Engineering Department

University Technology PETRONAS,

31750 Tronoh, Perak, Malaysia

Tel: +605-3687814, Fax: +605-3657443,

E-mail: nursyarizal_mnor@petronas.com.my

** Electrical and Electronics Engineering Department

University Technology PETRONAS,

31750 Tronoh, Perak, Malaysia

Tel: +605-3687833, Fax: +605-3657443,

E-mail:ramiah_j@petronas.com.my 


\section{Introduction:}

State Estimation (SE) is becoming increasingly important in modern energy management of power systems. Particularly with global deregulation of the power industry, power system state estimation has gained an even greater importance as a realtime monitoring tool [1], [2]. It is becoming a part and parcel of new energy control centre that are being established for large scale power systems. Its purpose is to establish a reliable and complete real time data base for on-line monitoring and control.

The complication of the interconnected power system due to new possibilities associated with open access and the operation of transmission networks, will cause the patterns of power flow in a deregulated power system become less predictable compared to the integrated systems of the past [3]. Hence, to achieve a more secure and economic operation, it is vital for utility operators to be properly informed of the operating condition or state of the power system.

The state of the power system is described by a collection of voltage vectors for a given network topology and parameters. Comprehensive discussion of the state of the art in electric power system state estimation is discussed in [1], [4] and [5]. The state variables obtained from $\mathrm{SE}$ is relying on the set of measurements that collected via the Supervisory Control and Data Acquisition (SCADA) system. The estimation process can be carried out through two functions of stochastic approach namely state forecasting and state filtering. State forecasting uses the past information while state filtering determines the optimal estimate by considering all available measurements and predicted states.

In state filtering various numerical methods of SE are presented in [1] and [6][14]. The objective of those methods is to have a robust numerical estimator, which can suitably improve the gain matrix. Recently, Artificial Intelligent system such as Fuzzy and Neural Network is started to use in SE [15]-[17]. However those techniques are not yet tested on the large scale power system.

Most of the approaches in SE is valid only for over-determine systems. Sufficient measurements are necessary to use those methods. However, in real time environment, some measurements are inconsistent in producing a true reading. The uncertainty in analog measurements could occur because of the combination between systematic error and random error [18]. Large systematic errors can be reduced by using appropriate calibration while the random errors will always remain and will influence the accuracy of estimated state [18]. Nevertheless, the small random errors can be filtered out using a proper weight to the set of measurements. An accurate measurement is weighted more than a less accurate. Decisions to select the good measurements are always based on the experience of engineers. Meanwhile, the measurement weights are assigned based on some assumed accuracy of the measuring instruments. 
However, the measurements that are assigned with high weightage need not be a good data. All the measurements need to be scanned first before processing SE. Typically most of the commercial software perform a pre-screening process to check whether the measured values are within the reasonability limit or not. The margin of the limitations set in the SCADA subsystem is typically around $10 \%$. However, for a large scale power system the pre-screening process is not fully screen or filter. The problem of accuracy will occur if the good measurements with a high weighting factor suddenly turn to bad measurements. An improved algorithm, which adaptively updates measurement variances, is presented in [19] and [20]. The sensitivity relationship between the measurement variances and the covariance matrix of their residuals was used for this purpose. However, the requirement of all the elements in the sensitivity matrix however could increase the cost since it calls for very large memory. In this paper a new approach to filter and forecast the state variable is proposed. This method uses Autoregressive (AR) and is known as Modified Covariance (MC) method.

\section{Review of Weight Least Square:}

Most SE programs in practical use are formulated as over-determined systems of nonlinear equations and solved as Weight Least Square (WLS) problems [1], [9]. In WLS method the measured quantities are represented as sum of true values and errors as

$$
\begin{aligned}
& z=z_{\text {true }}+e \\
& e=z-z_{\text {true }}=z-H x
\end{aligned}
$$

where ' $e$ ' represents the errors between the actual measurements $z$ and the true values $z_{\text {true }}(\mathrm{Hx})$ of the measured quantities. ' $x$ ' is the true value of the state variables. The true values cannot be determined, but their estimates $\hat{x}$ can be determined. The estimated error is given by

$$
\hat{e}=z-\hat{z}=z-H \hat{x}=e-H(\hat{x}-x)
$$

The objective function to be minimized is

$$
f=\sum_{j=1}^{m} w_{j} e_{j}^{2} \text { or } \sum_{j=1}^{m} \frac{1}{\sigma_{j}^{2}} e_{j}^{2}
$$

where ' $w$ ' is the weighting factor for the respective measurement. The standard deviation, $\sigma_{j}$, can be derived from the defined instrument errors on potential transformer (PT), current transformer (CT), transducer (XDUCER), Analog to Digital Converters (A/D) and the number of standard deviations assigned to the maximum possible error for a measurement (SIGMA) [21]. Thus, 


$$
\sigma=\frac{(P T+C T+A / D+X D U C E R)}{100 \bullet \text { SIGMA }} \bullet \text { base }
$$

Typically SIGMA is equivalent to 3.0 which determine that three standard deviations include $99.74 \%$ of the area under a normal distribution curve.

The best estimate is the one which makes the objective function ' $f$ ' to take on its minimum value. This is achieved when

$$
\frac{\partial f}{\partial x}=\sum_{j=1}^{m} 2 w_{j} e_{j} \frac{\partial e_{j}}{\partial x_{j}}=0
$$

The partial derivates with respect to state variables are the elements of the Jacobian matrix. Using the matrix notations of equation (2.2) in equation (2.5) yields

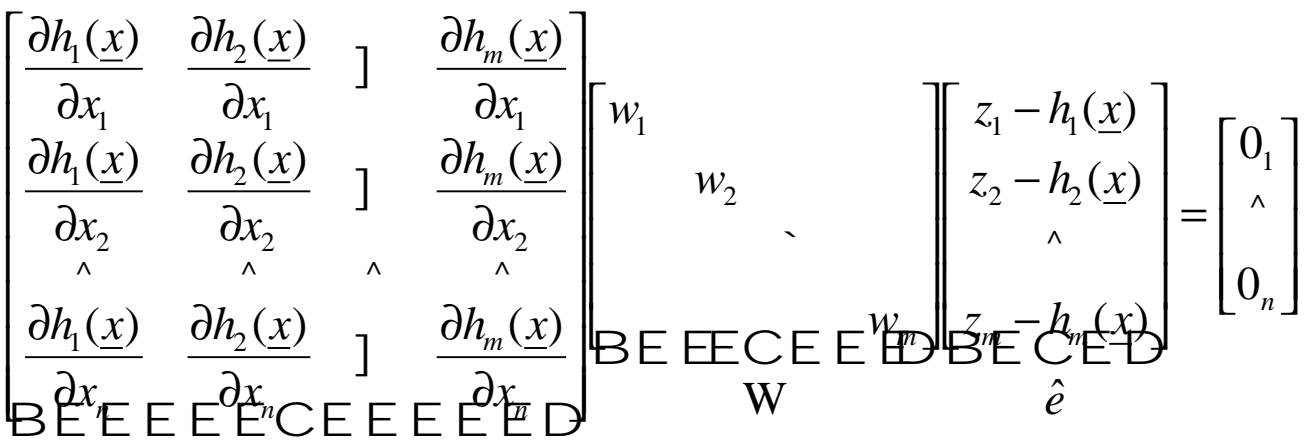

$$
\begin{aligned}
& \mathrm{H}_{x}^{T}
\end{aligned}
$$

Newton's method is used to solve this equation. The function $\underline{h}(\underline{x})$ has to be linearised at the initial value $\underline{x}^{(0)}$. Thus

$$
\begin{aligned}
& \underline{h}(\underline{x})=\underline{h}\left(\underline{x}^{(0)}\right)+\mathrm{H}_{x}^{(0)} \Delta \underline{x}^{(0)} \\
& \Delta \underline{x}=\underline{x}^{(1)}-\underline{x}^{(0)}
\end{aligned}
$$

Simplifying further, in $k$ th iteration, state variables are calculated as shown in equation (2.8) [22].

$\left.\left[\begin{array}{c}x_{1}^{(k+1)} \\ \mathrm{M} \\ x_{m}^{(k+1)}\end{array}\right]-\left[\begin{array}{c}x_{1}^{k} \\ \mathrm{M} \\ x_{m}^{(k)}\end{array}\right]=\underset{\mathbf{1} 4 \mathrm{H}^{T} \underline{\mathrm{W}}^{T} \mathrm{~W}}{\mathrm{G}}\right)^{-1} \mathrm{H}_{x}^{T} \mathrm{~W}\left[\begin{array}{c}z_{1}-h_{1}\left(\underline{x}^{(k)}\right) \\ \mathrm{M} \\ z_{m}-h_{m}\left(\underline{x}^{(k)}\right)\end{array}\right]$

If the gain matrix, $\mathrm{G}$, is non-singular, equation (2.8) gives estimated state variables. If there is lack of sufficient measurements, then $G$ is not invertable. 


\section{$\underline{2.1 \text { Bad Data Identification and Elimination }}$}

Methods used for detecting and identifying bad data are discussed in details in [10],[22]-[25]. The true measurement error is unknown, but it can be replaced in the objective function by an estimate. The objective function itself is a random variable which has a probability distribution area. In order to use those areas, we need to know the mean value of the objective function. Equation (2.9) expresses that the expected value of objective function is numerically equal to the number of degrees of freedom. It is also called the measurement redundancy scheme.

$$
E[\hat{f}]=E\left[\sum_{j=1}^{N_{m}} \frac{\hat{e}_{j}^{2}}{\sigma_{j}^{2}}\right]=\sum_{j=1}^{N_{m}} \frac{R_{j j}^{\prime}}{\sigma_{j}^{2}}=N_{m}-N_{s}
$$

$R^{\prime}$ in the above equation is a measurement error matrix, whose diagonal terms are the error variances. It can be obtained from equation (2.10).

$$
\mathrm{R}^{\prime}=\mathrm{R}-\mathrm{H} \mathrm{G}^{-1} \mathrm{H}^{\mathrm{T}}
$$

The weighted square sum of object function has the Chi-square, $\chi^{2}$, distribution. If the sum of weighted squares as calculated from the equation (2.4) satisfies the inequality $\left(\hat{f}<\chi_{k, \alpha}^{2}\right)=(1-\alpha)$, then the estimated values are accepted as the final estimates [10], [25]. If not, the variable with the largest standardized error $\left(\hat{e}_{j} / \sqrt{R_{i j}^{\prime}}\right)$ is eliminated and the states are re-estimated.

\section{Stochastic Methods:}

State estimation problems can be classified as over-determined, completely determined or under-determined depending on whether $N_{m}>N_{s}, N_{s}=N_{m}$ and $N_{m}<N_{s}$ respectively where $N_{m}$ is the number of measurements and $N_{s}$ is the number of state variables. A unique solution is possible only for over-determined and completely determined systems. SE techniques are traditionally performed on an over-determined system where the number of rows in $H$ exceeds the number of columns. If the covariances of the measurements are known, it can be used to place different weights on the various measurements. In practice the weights are assigned by based on experience and technical knowledge of engineers. However, if the measurement covariances are unknown, then $R$ is simply replaced by an identity matrix, i.e. uniform weights are assigned for all the measurements.

However, the technical challenge in SE is to solve under-determined systems of equations. In these systems, a unique solution may not exist, that is it is not solvable or $\mathrm{SE}$ is known as unobservable. However this can be solved if we add more state 
information.

As in state forecasting, it is possible to use previous data or historical data. The insufficient number of measurement can be compensated if a proper method is proposed. Therefore in this paper the MC method is used in order to forecast the data and at the same time filtering the logical weighting factor that has been assigned to the trusted measurements.

\subsection{Autoregressive Method}

In a $m$-th order AR process the signal $\mathrm{x}(\mathrm{n})$ is described by a weighted sum of preceding signal values plus an independent identically distributed noise signal, $\varepsilon(n)$ with variances $\sigma^{2}$ :

$$
\varepsilon_{m}(n)=x(n)+\sum_{k=1}^{m} a_{m}(k) x(n-k)
$$

where $a_{m}(k)$ are the prediction coefficients with $0 \leq k \leq m-1, m=1,2, \ldots, p$.

The well known AR methods such as Yule Walker method, Burg method, Covariance method and also $\mathrm{MC}$ is aiming for minimizing the sum squares of the forward and backward prediction error.

$$
\varepsilon_{m}=\varepsilon_{m}^{+}+\varepsilon_{m}^{-}
$$

Although the aim is same, those methods have their own advantages and disadvantages [26]. Due to the ability to minimize the sum of the squares errors in randomly, the MC is selected as the AR method in this paper.

\subsubsection{Modified Covariance method}

To derive the estimator, suppose that we are given the data $x(n), n=0,1, \ldots, N-1$, and let us consider the forward and backward linear prediction estimates of order $m$, as

$$
\begin{array}{r}
\hat{x}(n)=-\sum_{k=1}^{m} a_{m}(k) x(n-k) \\
\hat{x}(n-m)=-\sum_{k=1}^{m} a_{m}^{*}(k) x(n+k-m)
\end{array}
$$

and the corresponding forward and backward errors $f_{m}(n)$ and $g_{m}(n)$ as $f_{m}(n)=x(n)-\hat{x}(n)$ and $g_{m}(n)=x(n-m)-\hat{x}(n-m)$. The least square error is 


$$
\varepsilon_{m}=\sum_{n=m}^{N-1}\left[\left|f_{m}(n)\right|^{2}+\left|g_{m}(n)\right|^{2}\right]
$$

To find the prediction coefficients that minimize $\varepsilon_{m}$, the derivative of $\varepsilon_{m}$ with respect to $a_{m}^{*}(l)$ equal to zero for $l=1,2, \ldots . ., m$. Hence

$$
\begin{aligned}
\frac{\partial \varepsilon_{m}}{\partial a_{m}^{*}(l)} & =\sum_{n=m}^{N-1}\left[f_{m}(n) \frac{\partial\left[f_{m}(n)\right]^{*}}{\partial a_{m}^{*}(l)}+\left[g_{m}(n)\right]^{*} \frac{\partial g_{m}(n)}{a_{m}^{*}(l)}\right] \\
& =\sum_{n=m}^{N-1}\left[f_{m}(n) x^{*}(n-l)+\left[g_{m}(n)\right]^{*} x(n-m+l)\right]=0
\end{aligned}
$$

Substituting equation (3.3) to (3.5) into equation (3.6) and simplifying we find that the normal equation for the MC method are given by

$$
\begin{aligned}
& \sum_{k=1}^{m}\left[c_{x}(l, k)+c_{x}(m-k, m-l)\right] a_{m}(k) \\
& =-\left[c_{x}(l, 0)+c_{x}(m, m-l)\right]
\end{aligned}
$$

where $c_{x}(l, k)=\sum_{n=m}^{N-1} x(n-k) x^{*}(n-l)$ and known as autocorrelation coefficients, which dependent only on the absolute value of the difference between $l$ and $k$, i.e. $c_{x}(l, k)=c_{x}(|l-k|)$. However the autocorrelation matrix is not Toeplitz but it is symmetric.

In this paper, number of sample of previous data of measurements is taken from simulated results of load flow on the IEEE 24-bus network. The number of order is chosen is 2. Sample of voltage data at bus 2 is shown in Figure (1). All the measured quantities are taken for each bus. 


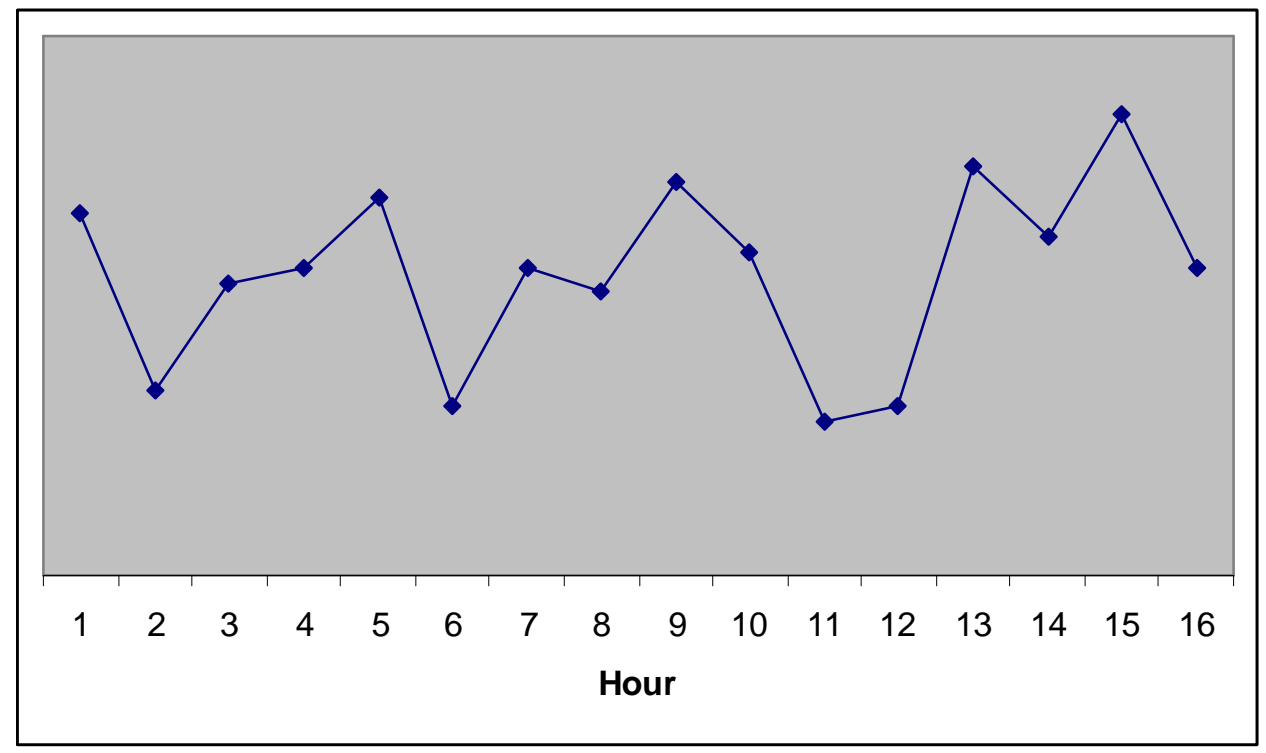

Figure (1): Voltage at bus 2 for 24 hours data.

\section{Component of $S E$}

The complete scheme that involved in SE algorithm is shown in Figure (2). The Measurement Data Plausibility Checking is used to detect and separate out all measurements with some apparent error in order to avoid any heavy distortion of the estimated network state due to completely wrong measurements. The proposed MC algorithm will is applied in this component. It will filter and forecast all the measurements prior to using them as input for SE. Those measurements with high weightage will be checked for its accuracy. If the related measurement shows an error more than 5\%, the weightage will be reduced to cater with its accuracy.

The function of a topology processor is to deliver an updated, consistent model of the system in terms of topology and measurements, based on known system connections and parameters and real-time SCADA input. It must divide the network into electrical islands if necessary, provide the necessary system parameters, and place measurements in their topological locations. It may also serve other important but less essential functions, such as posting warnings for circuit breaker and/or isolator.

Instead of numerical and hybrid methods, topological is the main approache for Observability [27]. After the topology of the network is checked, the network is checked for its observability. Generally, a region of the network is called observable, if the measurements in the system provide enough (non-redundant) information to estimate the state of that part of the network. 


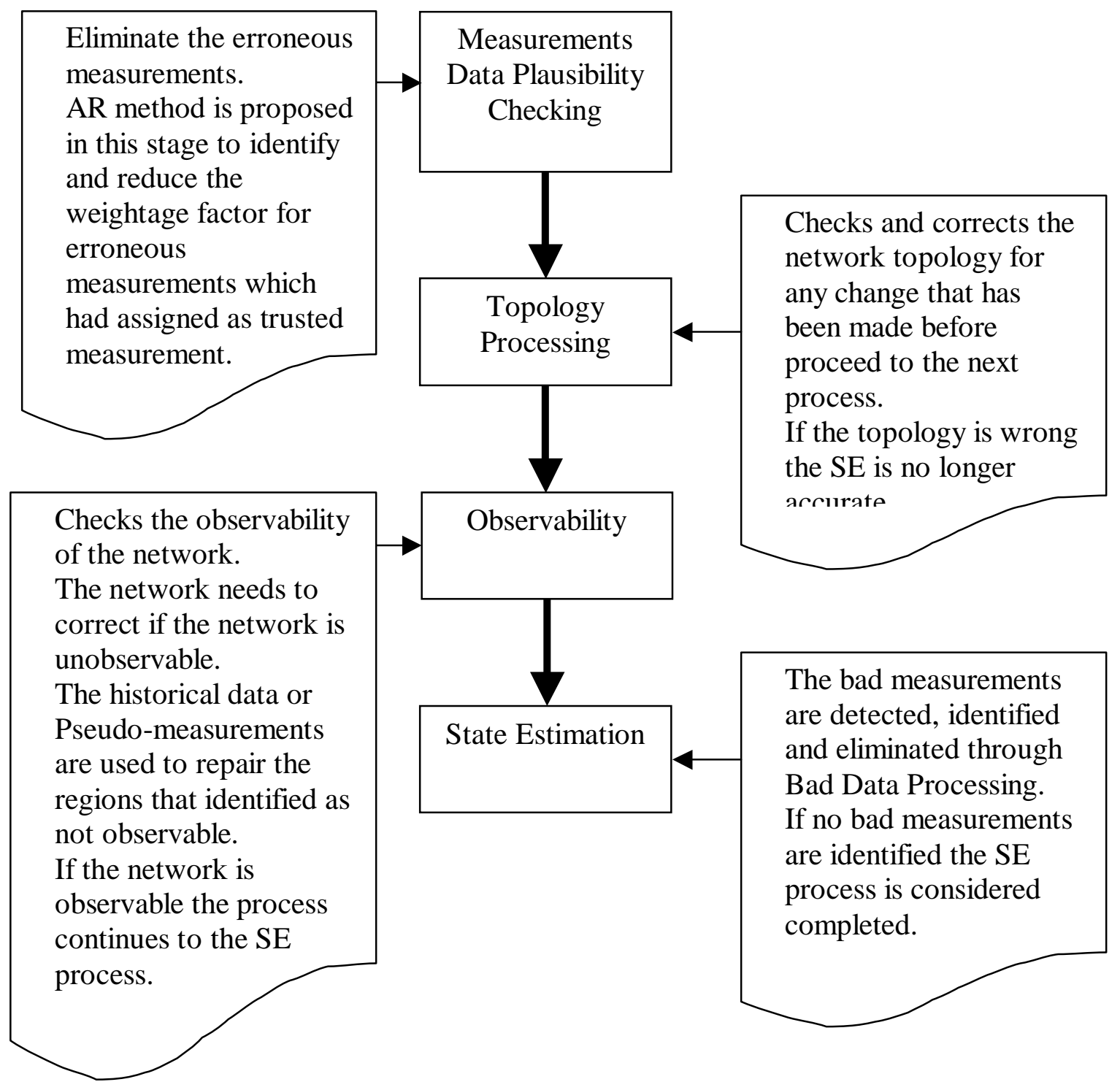

Figure (2): The scheme that involved in SE algorithm.

\section{Numerical Study:}

For case study IEEE 24-bus network is chosen. Three case studies are carried out. In Case 1, under-determined system is tested, while Case 2 will consider a notconvergent system due to the incorrect assigning of weight factor. In Case 3 the system is tested for detecting the weighting factor for erroneous measurements assigned with high weighting.

\section{Case 1}

The number of states is 47 while number of measurements is 41 . As per definition the system is known as under-determined system. The network becomes unobservable. 
The proposed method is able to forecast and use the forecasted value as an input for SE.

Table (1) shows the value of actual, forecasted and estimated values.

Table (1): The actual, forecasted and estimated values of state variables - Case 1

\begin{tabular}{|l|l|l|l|l|l|}
\hline \multicolumn{2}{|l|}{ Actual } & \multicolumn{2}{l|}{ Modified Cov. } & \multicolumn{2}{l|}{ Estimated } \\
\hline $\mathrm{V}(\mathrm{pu})$ & $\theta(\mathrm{rad})$ & $\mathrm{V}(\mathrm{pu})$ & $\theta(\mathrm{rad})$ & $\mathrm{V}(\mathrm{pu})$ & $\theta(\mathrm{rad})$ \\
\hline 1.035 & 0.000 & 1.036 & 0.000 & 1.036 & 0.000 \\
\hline 1.035 & -0.031 & 1.036 & -0.031 & 1.036 & -0.032 \\
\hline 0.961 & -0.213 & 0.962 & -0.213 & 0.962 & -0.203 \\
\hline 0.968 & -0.216 & 0.969 & -0.215 & 0.969 & -0.216 \\
\hline 0.983 & -0.176 & 0.984 & -0.175 & 0.984 & -0.166 \\
\hline 0.965 & -0.306 & 0.966 & -0.305 & 0.966 & -0.296 \\
\hline 1.025 & -0.324 & 1.026 & -0.323 & 1.026 & -0.314 \\
\hline 0.972 & -0.377 & 0.973 & -0.376 & 0.973 & -0.367 \\
\hline 0.967 & -0.290 & 0.968 & -0.289 & 0.968 & -0.291 \\
\hline 0.984 & -0.301 & 0.985 & -0.300 & 0.985 & -0.291 \\
\hline 0.965 & -0.250 & 0.966 & -0.249 & 0.966 & -0.240 \\
\hline 0.971 & -0.225 & 0.972 & -0.224 & 0.972 & -0.215 \\
\hline 0.965 & -0.247 & 0.966 & -0.246 & 0.966 & -0.237 \\
\hline 0.980 & -0.207 & 0.981 & -0.206 & 0.981 & -0.197 \\
\hline 1.014 & 0.001 & 1.015 & 0.001 & 1.015 & 0.011 \\
\hline 1.017 & -0.020 & 1.018 & -0.020 & 1.018 & -0.010 \\
\hline 1.038 & 0.054 & 1.039 & 0.055 & 1.039 & 0.064 \\
\hline 1.050 & 0.076 & 1.051 & 0.076 & 1.051 & 0.086 \\
\hline 1.022 & -0.056 & 1.023 & -0.056 & 1.023 & -0.046 \\
\hline 1.038 & -0.050 & 1.039 & -0.049 & 1.039 & -0.040 \\
\hline 1.050 & 0.093 & 1.051 & 0.094 & 1.051 & 0.103 \\
\hline 1.050 & 0.191 & 1.051 & 0.192 & 1.051 & 0.201 \\
\hline 1.050 & -0.032 & 1.051 & -0.032 & 1.051 & -0.022 \\
\hline 0.979 & -0.078 & 0.980 & -0.077 & 0.980 & -0.068 \\
\hline & & & & & \\
\hline
\end{tabular}

The average error in MC method is $0.069 \%$ for the voltage magnitudes and 2.983 $\%$ for the voltage phase angles. The summary results of SE are shown in Table (2). The SE runs twice in this case. The first estimate of SE gives an output of unobservable due to $N_{m}<N_{s}$ condition. The second estimate processes the input of SE taken from MC's output and the SE is successful to converge in 3 iterations with a tolerance of 0.001 and $N_{m}$ is equal to 148 . 
Table (2): State Estimator summary results - Case 1

\begin{tabular}{|l|l|l|l|}
\hline $\begin{array}{l}\text { Est. } \\
\text { No }\end{array}$ & Weight sum of square, $\hat{f}$ & Chi-square distribution, $\chi_{k, a}^{2}$ & Iter. \\
\hline 1 & - & - & Unobservable \\
\hline 2 & 8.3495 & 125.4584 & 3 \\
\hline
\end{tabular}

\section{Case 2}

The convergence of SE normally relies on the tolerance, the number of measurements and the weighting factors assigned to the individual measurements. In this case study, the network is adjusted to be not converging by changing the weighting the factors. In the first run of the SE, the result is not converging when the tolerance, number of measurements and maximum number of iteration are $0.001,117$ and 50 respectively.

When MC algorithm is introduced in the SE process, it is able to solve the problem taking all the predicted values, increasing the number of measurements to 148 and the weighting factor also increased in number related with number of measurements. The results of state variables obtained after estimated and forecasted are depicted in Table (1).

The average error in MC method is $0.069 \%$ for the voltage magnitudes and 2.983 $\%$ for the voltage phase angles. The summary results of SE are shown in Table 3. The $\mathrm{SE}$ is run twice in this case where for the first estimate SE gives an output of "not converging'. The second estimate processes the input of SE taken from MC's output and the SE is successful in getting the results converged at iteration 3 for a tolerance of 0.001 and $N_{m}$ of 148 .

Table (3): State Estimator summary results - Case 2

\begin{tabular}{|l|l|l|l|}
\hline $\begin{array}{l}\text { Est. } \\
\text { No }\end{array}$ & Weight sum of square, $\hat{f}$ & Chi-square distribution, $\chi_{k, a}^{2}$ & Iter. \\
\hline 1 & - & - & $\begin{array}{l}\text { Not converge } \\
\text { Iteration set to 50 }\end{array}$ \\
\hline 2 & 8.3522 & 125.4584 & 3 \\
\hline
\end{tabular}

\section{Case 3}

In this case study, 5 bad measurements are introduced. The directions of P8, Q8, P2-6 and Q5-10 are reversed, while reading of V9 increased to $1 \mathrm{pu}$. In the first run of $\mathrm{SE}$, the MC algorithm detected a few analog measurements with wrong readings compared with forecasted value, typically more than $5 \%$ error, as depicted in Table (4). After changing the assigned weight factor for the identified measurements with lower value, the SE result obtained are shown in Table (5). This shows that the program 
correctly detects, identifies and eliminates the measurements errors.

Table (4): MC's result - Case 3

\begin{tabular}{|l|l|}
\hline Analog measurements with more than 5\% error & $\mathrm{V} 9, \mathrm{P} 8, \mathrm{Q} 8, \mathrm{P} 2-6, \mathrm{Q} 5-10$ \\
\hline $\begin{array}{l}\text { Analog measurements considered bad and the weight } \\
\text { factor is high. }\end{array}$ & $\mathrm{V} 9$ \\
\hline
\end{tabular}

Table (5): State Estimation results - Case 3

\begin{tabular}{|l|l|l|l|l|}
\hline $\begin{array}{l}\text { Est. } \\
\text { No }\end{array}$ & $\begin{array}{l}\text { Bad } \\
\text { Data }\end{array}$ & Weight sum of square, $\hat{f}$ & Chi-square distribution, $\chi_{k, a}^{2}$ & Iter. \\
\hline 1 & Q5-10 & $1.34 \mathrm{E}+03$ & $9.05 \mathrm{E}+01$ & 11 \\
2 & V9 & $9.33 \mathrm{E}+02$ & $8.94 \mathrm{E}+01$ & 7 \\
3 & P2-6 & $5.69 \mathrm{E}+02$ & $8.83 \mathrm{E}+01$ & 7 \\
4 & Q8 & $2.61 \mathrm{E}+02$ & $8.71 \mathrm{E}+01$ & 7 \\
5 & P8 & $1.46 \mathrm{E}+02$ & $8.60 \mathrm{E}+01$ & 7 \\
6 & None & $4.72 \mathrm{E}+00$ & $8.48 \mathrm{E}+01$ & 3 \\
\hline
\end{tabular}

\section{Conclusions:}

The results of Case 1 and Case 2 clearly show that the observability and non converging problems of SE can be solved if the MC method is added in SE process. Table (1) depicted that the errors in voltage phase angles, between forecasted value and the estimated value are small. Further, in Case 3, the results show that the proposed method enables to list all the bad measurements that were initially assigned with high weight factor. The results also show that the SE is capable to identify and eliminate the bad measurements after the weight factor is reduced for those bad measurements with high weight factor. Thus the strength of MC algorithm in the field of SE is established. 


\section{References:}

[1] A. Monticelli, "Electric Power System State Estimation", Proc. IEEE, Vol. 88, pp. 262-282, Feb. 2002.

[2] Wu, F.F., Moslehi, K., Bose, A., "Power System Control Centers: Past, Present, and Future", Proc. IEEE, Vol. 93, pp. 1890-1908, Nov. 2005.

[3] A.P. Sakis Meliopoulus, Bruce Fardanesh and Shalom Zelingher, "Power System Estimation: Modeling Error Effects and Impact on System Operation", IEEE Systems Science, pp. 682-690, January, 2001.

[4] A. Bose and K. A. Clements, "Real-time Modeling of Power Networks", Proc. IEEE, Vol. 75, pp. 1607-1622, Dec. 1987.

[5] F. F. Wu, "Power System State Estimation: Survey", International Journal Elect. Power Eng. System, Vol. 12, pp. 80-87, Jan. 1990.

[6] Holten, L.; Gjelsvik, A.; Aam, S.; Wu, F.F.; Liu, W.-H.E, "Comparison of different methods for state estimation", IEEE Trans on Power System, Vol. 3, No 4, pp. $1798-1806,1988$.

[7] Simoes Costa, A.; Piazza, T.S.; Mandel, A., "Qualitative methods to solve qualitative problems in power system state estimation", IEEE Trans on Power System, Vol. 5, No 3, pp.941 - 949, 1990.

[8] Al-Othman, A.K.; Irving, M.R.;A., "Comparative study of two methods for uncertainty analysis in power system State estimation", IEEE Trans on Power System, Vol. 20, No 2, pp. 1181 - 1182, 2005.

[9] F. C. Schweppe and E. J. Handschin, "Static state estimation in electric power systems”, Proc. IEEE, Vol. 62, pp. 972-983, 1974.

[10] Ali Abur and Antonio Gomez Exposito, "Power System Estimation: Theory and Implementation", Marcel Dekker, Inc., 2004.

[11] J. W. Wang and V. H. Quintana, "A decoupled orthogonal row processing algorithm for power state estimation," IEEE Trans Apparatus and System, pp. 2337-2344, 1984. 
[12] A. Simoes-Costa and V. H. Quintana, "A robust numerical technique for power system state estimation", IEEE Trans Apparatus and System, Vol.100, pp. 691698, 1981.

[13] A. Simoes-Costa and V. H. Quintana, "An orthogonal row processing algorithm for power system sequential state estimation", IEEE Trans Apparatus and System, Vol. 100, pp. 3791-3800, 1981.

[14] J. A. George and M. T. Heath, "Solution of sparse linear least squares problems using Givens notations", Linear Algebra and Appl., Vol. 34, pp 69-83, 1980.

[15] Mohammad Shahidehpour and Muhammad Marwali, "Role of Fuzzy Sets in Power System State Estimation", International Journal of Emerging Electric Power Systems, The Berkeley Electronic Press (bepress), Vol.1, Issue 1, Article 1003, 2004.

[16] Jorge Pereira, Vladimiro Miranda and J. Tome Saraiva, "Fuzzy Control of State Estimation Robustness", Proceedings of 14 ${ }^{\text {th }}$ PSCC, Paper 5, June 2002.

[17] Sami Repo and Juhani Bastman, "Applicability of Neural Network in Power System Computation", Report for Power Engineering Group, Tampere University of Technology, Tampere, Finland, 1996.

[18] M.M. Adibi and R. J. Kafka, "Minimization of Uncertainties in Analog Measurements For Use in State Estimation", IEEE Transactions on Power, Vol.5, No. 3, pp. 902-910, 1990.

[19] G. Liu, "Novel algorithms to estimate and adaptively update measurement error variance using power system state estimation results", Electric Power System .Research, Vol. 47, No. 1, pp. 57-64, Oct. 1998.

[20] Shan Zhong and Ali Abur, "Auto Tuning of Measurement Weights in WLS State Estimation”, Transactions on Power Systems, Vol. 19, No. 2, pp. 2006-2013, 2004.

[21] Tenaga Nasional Berhad EMS Staff, "Network Analyst's Guide”, ESCA Corporation, 1994.

[22] John J. Grainger and William D. Stevenson, Jr., "Power System Analysis", McGraw-Hill InternationalEditions, 1994. 
[23] E. J. Handschin, F. C. Schweppe, J. Kohlas and A. Fiechter, "Bad Data Analysis for Power Systems State Estimation", IEEE Trans Apparatus and System, Vol. 94, pp. 329-337, 1975.

[24] T. V. Cutsem and M. Ribbens-Pavella, "Critical Survey of Hierarchical Methods for State Estimation of Electrical Power Systems," IEEE Trans. On Power Apparatus and Systems, Vol. 102, No.10, pp. 3415-3424, October 1983.

[25] A. Monticelli, "State Estimation in Electric Power Systems. A Generalized Approach”, Kluwer Academic Publishers, 1999.

[26] M. H. Hayes, "Statistical Digital Signal Processing and Modeling", John Wiley \& Sons, Inc., 1996.

[27] A. Monticelli, Wu FF, "Network Observability: Identification of Observable Islands and Measurement Placement", IEEE Trans Power Apparatus Systems, Vol. 104, No. 5, pp 1035-1041, 1985.

\section{Nomenclatures:}

$\begin{aligned} \sigma & \text { Standard Deviation } \\ \chi^{2} & \text { Chi-square } \\ e & \text { Error } \\ w & \text { Weighting Factor } \\ z & \text { Measurements } \\ \mathrm{G} & \text { Gain } \\ \mathrm{H} & \text { Jacobian Matrix } \\ N_{s} & \text { Number of states } \\ N_{m} & \text { Number of measurements } \\ \varepsilon & \text { Least square error }\end{aligned}$

